\title{
Contra la marginalidad historiográfica
}

doi: http://dx.doi.org/10.32870/espiral.v24i69.6730.g5857

Elisa Cárdenas Ayala*

El delito y la marginalidad, después de haber sido objetos recurrentes de la preocupación de las ciencias sociales decimonónicas en estrecho vínculo con el interés de todo tipo de Gobiernos por el mantenimiento del orden social, son en la actualidad parte de un campo sólido de la investigación histórica, en diálogo con preocupaciones de la sociología y de la antropología contemporáneas. Los estudios sobre la memoria, bastante más recientes, pues se tornaron centrales en la segunda mitad del siglo xx, ocupan también un espacio propio no sólo en historia, sino en las ciencias sociales en general, como una forma de combatir, desde la academia, las políticas del olvido características del sistema capitalista contemporáneo.

En Voces y memorias del olvido. Historia marginalidad y delito en América Latina, libro coordinado por Jorge Alberto Trujillo Bretón, y publicado en México por la Universidad de Guadalajara en el año 2015, se entrecruzan de manera fructífera estos dos campos, contribuyendo a mostrar las posibilidades múltiples de una historia social en donde el tema de la memoria ocupa un lugar central, por lo que se sitúa en diálogo con

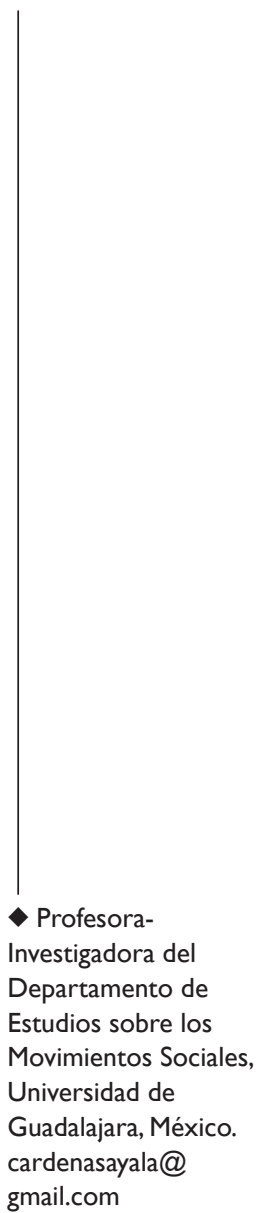
heridas constitutivas de nuestras sociedades contemporáneas. El libro, que reúne quince capítulos y una presentación a cargo

Jorge Alberto Trujillo Bretón (coord.) (20I5). Voces y memorias del olvido. Historia marginalidad y delito en América Latina. Guadalajara: Universidad de Guadalajara. 
del coordinador, nace de un esfuerzo colectivo: la creación de la Red de Historiadores e Historiadoras de las Américas (Redhhda), luego del éxito de una reunión académica llevada a cabo en mayo de 2011 en la Universidad de Guadalajara, México (el I Coloquio de Historia, Marginalidad y Delito en América Latina, con participantes de diversos países del continente).

Los dos capítulos con que inicia el volumen ofrecen una reflexión teórica y metodológica, esfuerzo del que participa (aunque en un espacio limitado) la introducción, a cargo del coordinador de la obra, que subraya las aristas principales del campo, los retos de la investigación y algunos logros recientes. Los trece capítulos restantes, que constituyen el grueso de la obra, son producto de investigaciones originales de sus autores, en donde la reflexión se edifica a partir de la exploración de materiales de archivo, entrevistas y revisión bibliográfica acuciosa. Los textos tienen en común el poder ser leídos con agilidad-algunos haciendo gala de excelente pluma-. El libro logra presentar un perfil coherente, articulado a partir del mirador que constituye el vínculo entre delito y marginalidad en diversas de sus facetas posibles. Así, en un conjunto en que destaca el interés por los sectores populares, se ofrecen acercamientos en perspectiva histórica sobre una variedad de casos al sistema de justicia y al sistema penal, a la situación de los sujetos frente a la justicia, así como al funcionamiento de los sistemas represivos, con énfasis en los sistemas carcelarios, en su teoría, en su práctica y en su materialidad espacial.

También se proponen análisis de las formas en que en la construcción social del estigma -caracterizado hace ya tiempo y finamente por Goffman- se cruzan, entre otros factores, clase social y género. El mismo conjunto ofrece exploraciones de la racionalidad presente en la definición de conductas estigmatizadas por la sociedad y la ley, así como en las formas de relación de actores individuales y colectivos 
con el orden jurídico y legal de su tiempo, en algunos casos como estrategias de resistencia frente al orden impuesto.

También es central en el volumen la atención al discurso relacionado con el mundo del crimen y de su represión, sea por parte de los estudiosos del tema en su momento, de las autoridades, o de la prensa, así como de los sujetos implicados directamente en la acción delictiva. Así, Voces $y$ memorias del olvido... ofrece un conjunto de miradas sobre sociedades cuyo común denominador es un ideal de "modernidad" perseguido por las élites, en función del cual construyen un orden institucional que se acompaña de la represión de ciertas conductas previa construcción acuciosa de estigmas y culpabilidades.

El conjunto de los casos trabajados cubre un periodo largo, que se extiende de finales del siglo XVIII hasta mediados del siglo XX, si bien el siglo XIX es el que concentra la mayoría de los estudios. Los capítulos llevan al lector por diversos escenarios de la geografía iberoamericana (y no sólo latinoamericana, contra lo que el título anuncia): de México, el país más representado en los estudios y al que corresponden también los capítulos dedicados a periodos más tempranos, se abordan casos ubicados en los actuales estados de Chiapas, Sinaloa, Jalisco, Veracruz, Puebla y Baja California Norte; un capítulo nos acerca al caso del Uruguay y otro a Chile; y uno más, único esfuerzo comparativo entre países en el volumen, da cuenta de la expansión, apogeo y declive del modelo del panóptico en los sistemas penitenciarios iberoamericanos, con lo cual el lector puede cruzar por un momento el Atlántico para situarse en varios puntos de la geografía de la España contemporánea.

Para los interesados en el tema, el volumen ilustra prolijamente la diversidad de fuentes que permiten abordar su estudio, más allá de los archivos judiciales que en los años 80 empezaran a ser objeto de interés de historiadores ahora muy reconocidos como Carlo Ginzburg y Arlette Farge, a 
quienes se deben importantes aportaciones a la historia social en las temáticas que son centrales dentro de la obra que aquí se comenta.

Como en todo libro colectivo, hay diferencias en la madurez de los textos presentados, en cuyo detalle no entraré aquí para no abusar de la atención del lector, pero son diferencias que, entre otras cosas, sin duda obedecen a las trayectorias diversas de los participantes, mismas que podemos intuir pero que desafortunadamente desconocemos por hacer falta en el libro indicaciones uniformes sobre los distintos autores. No quiero, sin embargo, dejar de resaltar por bien logrados los textos de Rocío Ortiz Herrera, Rosalina Estrada Urroz y Eric Michael Schantz, que en lo personal me resultaron sumamente sugerentes.

Cabe subrayar la apuesta de iniciar el libro con reflexiones teóricas y metodológicas relativas a las dificultades que presenta el campo historiográfico, como el capítulo de Robert M. Buffington, "Cómo narrar la historia del delito en tiempos difíciles”, preocupado por las tensiones múltiples entre pasado y presente que habitan el trabajo del historiador (algo, es preciso insistir, común a todos los campos historiográficos), y por señalar lo que en su concepto son desafíos específicos que atañen a la escritura de la historia del delito. El autor presenta estos desafíos como relacionados con la comunicación de masas y con la espectacularidad del crimen dentro de lo que Guy Debord llamara la "sociedad del espectáculo". Invitando a desmitificar las fuentes y el análisis cuantitativo, Buffington denuncia: la estadística misma se vuelve espectáculo. El autor destaca la relación de la narración con su público, en cuyo fondo está la relación entre espectacularidad del crimen y sociedad civil, así como los retos que enfrenta el historiador en esa circunstancia que es propia de nuestro tiempo: "Hoy en día, el poder de los crímenes espectaculares y los pánicos morales no tiene rival en su capacidad de constituir a los públicos" (p. 36). Es 
este un texto que, apoyándose en W. Benjamin, plantea el deber del historiador no sólo de "recuperar el pasado, sino también de rescatarlo" (p. 37).

El segundo capítulo, que ofrece un acercamiento a la "Transformación del individuo en la sociedad moderna a partir de la Escuela de Frankfurt", de Mauricio F. Rojas Gómez, cumple también una función básica, para el lector, de acercamiento a elementos generales de comprensión del tema a partir de lo legado por una de las escuelas más influyentes en los estudios sociales en nuestra época, concretamente en la obra de Herbert Marcuse, Theodor Adorno y Max Horkheimer. Reconstruye de forma muy sugerente los planteamientos de estos autores sobre la relación entre el individuo (la persona) y la sociedad industrial, relación centrada en la tensión entre cultura e individualidad y en las transformaciones del alma, y la relación de estas con el individuo y la sociedad. Rojas Gómez formula una síntesis que merecería tener mayor eco en varios de los capítulos subsiguientes en la medida en que precisamente estas tensiones entre lo individual y lo colectivo habitan intensamente las lógicas modernas de la impartición de justicia, de la conservación o imposición del orden, de la normalización de las conductas, y aun de la memoria dolorosa (social, colectiva, individual) que estructuran el interés de la mayoría de los estudios reunidos en este libro.

Dos son los capítulos interesados por el orden colonial, y ambos abordan casos mexicanos. El primero de ellos, "Impartición de justicia y estrategias legalistas en pueblos zoques y tzotziles de Chiapas: del cabildo colonial al ayuntamiento constitucional, 1778-1857”, de Rocío Ortiz Herrera, se interesa por la transición entre el siglo XVIII y el XIX, entre las instituciones del llamado "antiguo régimen" y el orden republicano, misma que, entre otras cosas, representó el fin de un estatuto especial para la población indígena. La autora aborda la relación de Gobiernos indígenas (cabildos 
coloniales, ayuntamientos republicanos) con el sistema de justicia, a partir de los casos de los pueblos de Tecpatán y Copainalá, de la región zoque, y el pueblo tzoztil de Chamula, todos ellos en el actual estado de Chiapas. Explora el margen de autonomía construido por esos pueblos en materia de prácticas de justicia, transgrediendo "incluso las instituciones que los gobiernos de esos años establecieron para impartir justicia" (p. 71), margen de autonomía labrado en buena medida contra el poder colonial de la Iglesia católica y reflejado también (en el caso de Chamula) en materia religiosa; una confrontación que pasó en varios casos por la expulsión de los párrocos.

Ortiz Herrera estudia la práctica del poder político de los pueblos ejercido a través de sus ayuntamientos, práctica que caracteriza como basada en conceptos como el "bien común", no representados en la normatividad constitucional de las primeras décadas del régimen independiente. Es esta una contribución a la escritura de una historia muy marcada hasta nuestros días por el trabajo de los antropólogos y fortalecida en años recientes por contribuciones que muestran los múltiples recursos políticos y sociales de los pueblos frente a las formas de sujeción; temas que también han sido explorados para la historia del vecino estado de Oaxaca, y que enriquecen lo que sabemos sobre la participación en la política decimonónica de los pueblos indios y su fuerza local y regional.

El otro texto que se interesa por el fin del periodo colonial, de Betania Rodríguez Pérez, "Ebrios en Guadalajara durante el periodo colonial tardío, 1792-1821”, es producto de la exploración combinada de tres archivos mexicanos: el Archivo Histórico de la Real Audiencia de Guadalajara, el de la Arquidiócesis de Guadalajara y el Archivo General de la Nación. El capítulo rastrea el peso del estigma de la embriaguez en la sociedad de su tiempo, esbozando un perfil social de los detenidos por esa causa y por ende confrontados con 
la justicia, y ofrece una mirada particular sobre algunos de los casos que la autora consideró más relevantes dentro de un grupo de noventa expedientes encontrados. Muestra así un conjunto de comportamientos que fueron considerados desordenados en su tiempo: el estado mismo de embriaguez, el escándalo vinculado a ella y diversas formas de violencia, incluyendo la comisión de delitos, también asociadas a ella. El texto abre un conjunto de ventanas que sin duda podrían ser exploradas a mayor profundidad con ayuda de las mismas fuentes en temáticas diversas, que van desde el tipo de bebidas consumidas hasta la relación entre el estado de ebriedad y el tipo de sentencia impartida.

Se ofrece al lector, enseguida, como un capítulo bisagra, el texto de Miguel Ángel Isais Contreras, "La fama pública: un concepto útil para entender la sociedad y la justicia penal durante el siglo XIX", que muestra la forma en que este antiguo concepto atraviesa el siglo XIX perviviendo en el sistema penal mexicano y en las relaciones sociales, a pesar de que se constate una tendencia a diluirse su presencia. Siguiendo la que considera como una "pista jurídica para dar con la vida de los sectores populares" (p. 118), el autor muestra de qué manera la "mala fama", elemento básico de la justicia hispana presente al menos desde las Siete Partidas, fue parte de la administración de justicia novohispana y sobrevivió a la dominación política española para formar parte del derecho mexicano hasta finales del siglo XIX, a pesar de las severas críticas a su mantenimiento sostenidas por notorios jurisconsultos de la época. Lo más sugerente del texto, en mi opinión, es el vínculo que hace entre el combate al concepto de fama pública y la limitación de la función de los jurados populares, es decir, la búsqueda de "acotar la participación del pueblo dentro de la justicia" (p. 131), un tema en el que, sin embargo, desafortunadamente no se profundiza. 
Menciono que el texto de Isais Contereras se trata de un capítulo bisagra porque la fama pública, implicada ya en el estudio sobre los ebrios detenidos en Guadalajara, cumple una función importante también en el texto de Laura Benítez Barba, "Volverse a casar o casarse dos veces: bígamos en Jalisco, 1824-1860”. Este texto, que muestra mecanismos de colaboración entre la justicia civil y la religiosa, interpreta a la bigamia como un recurso frente a los límites que el sistema imponía a la vida de pareja en tiempos en que las uniones entre mujer y hombre eran consideradas para toda la vida. Muestra el rigor de los castigos a los infractores y la colindancia de delito y pecado en las prácticas de impartición de justicia, así como el poder social de la Iglesia, cuyo método de vigilancia moral más efectivo fue, según la autora, "la mirada de la comunidad" pues "vecinos, familiares y allegados tenían la obligación moral -como todo buen cristiano- de avisar de los actos contrarios a su fe" (p. 143).

Tras los hallazgos mostrados por la autora, que resaltan la centralidad del matrimonio como institución, la diferencia en la experiencia de la transgresión de la norma por parte de hombres y mujeres y la práctica de la justicia a partir de los casos concretos localizados en el archivo, se advierte la posibilidad de formular otras interrogantes que permitieran profundizar en el tema, como por ejemplo, qué tan representativas son de la valoración de la bigamia en la sociedad de su tiempo las denuncias encontradas pues, para un periodo que va de 1824 a 1860, la autora localizó dieciocho casos de denuncia por bigamia, ahí donde las disposiciones y los mecanismos represivos mostrados por el propio texto sugieren una práctica social extendida.

El tema de la reputación, que es el rostro social de la legal "fama pública", también acompaña al texto de Rosalina Estrada Urroz, "La pasión siempre culpable. El criminal y la víctima: una muestra de la prensa porfiriana". Es este un capítulo cuidadosamente elaborado, que estudia lo que 
ahora llamaríamos "sensacionalismo" a través de los casos de dos periódicos producidos en dos ciudades mexicanas: Veracruz y Puebla. De entrada, dice la autora, "El Presente y El Dictamen son receptáculos de una doble obsesión: retratar a la víctima en sus características más sobresalientes y al criminal en su fisonomía, actitudes y actos posteriores” (p. 157).

El texto se inspira en las reflexiones feministas de Joan W. Scott para explorar la relación entre historia y fantasía en la construcción de los relatos periodísticos sobre criminales y víctimas. Lo anterior lo hace considerando el contexto de la época y el marco interpretativo de los criminólogos mexicanos, que analiza siguiendo las huellas de autores mexicanistas reconocidos como Speckman y Picatto, pero también ampliando su perspectiva y acudiendo a la obra de estudiosos de otras latitudes y de otras disciplinas. Así, la autora propone paralelos entre expediente judicial y crónica, y destaca la tensión entre lo "real" y la fantasía, presentes en los relatos de una prensa caracterizada por la reproducción de estereotipos morales genéricamente marcados, que vehicula prejuicios de clase y muestra la segregación como rostro indisociable de la búsqueda de la civilización. Se trata de relatos influenciados por la literatura europea de la época, que contribuyen a perpetuar los estereotipos de la dudosa reputación de la víctima y el carácter nato del criminal. El capítulo se acompaña de un espectro bibliográfico amplio de la literatura sobre el tema, y su exploración de la construcción del relato, de los elementos que lo presiden, aunque de dimensiones mucho más acotadas, recuerda el fino estudio de Nicole Loraux sobre las formas que puede revestir el asesinato de las mujeres en la tragedia griega (Loraux, 1985).

El sugerente capítulo de Estrada Urroz es el primero de dos centrados en la prensa y en las lógicas de producción de su discurso sobre temas relacionados con la transgresión 
de la ley. El segundo, de la autoría de Luis Martín Padilla Ordoñes, se titula "Actividades delictivas en torno a las drogas en la prensa sinaloense, 1940-1960: influencias sobre su discurso". Este texto parte de una síntesis de la historia de la regulación y luego proscripción del consumo de sustancias que ahora conocemos comúnmente como "drogas", en el mundo occidental y específicamente en México, para argumentar que el discurso al respecto en la prensa sinaloense durante los años 1940-1960 estuvo directamente influenciado por el discurso gubernamental en la materia, vía que, considera el autor, llevó a que dicho discurso condenatorio se volviera "cotidiano en la opinión pública sinaloense a partir de la década de 1940” (p. 198).

El texto describe la vía de la construcción legal de una zona de delito que comprende todas las actividades relacionadas con la producción, distribución y consumo de estas sustancias, documentando a través de materiales de archivo, periódicos de la época y bibliografía especializada lo que la literatura crítica ha señalado desde hace varias décadas: el peso indubitable de los intereses y políticas estadounidenses en la definición de la postura de los Gobiernos mexicanos en materia de las llamadas "drogas", así como en el discurso de los medios mexicanos, desde los primeros años del "combate" contra su producción, uso y comercialización, muy señaladamente a partir de la posguerra de los años 40 .

Sobre el tema de sustancias de consumo controlado en México y la estigmatización de sus consumidores y comercializadores trata el capítulo de Eric Michael Schantz, "Surcando un hábito doméstico: la expansión interna del mercado de opiáceos y marihuana en México, 1936-1953”. Este capítulo, muy sugerente y bien escrito, es un ejercicio de historia social que explora la relación transfronteriza entre el crimen organizado y dicho mercado en la frontera entre México y los Estados Unidos, concretamente en Tijuana y Mexicali. "El caso de los enervantes", dice el autor, "ilustra 
cómo un cambio en el marco jurídico, tanto internacional como nacional, transformó a sujetos de origen humilde o socialmente marginados en objetos de persecución” (p. 221).

A través del tema de la adicción y de su represión por parte del Gobierno, el autor entrecruza la historia de la configuración urbana de estas ciudades, la de su relación con la frontera, con la migración extranjera (especialmente la colonia china) y el funcionamiento de prácticas a la vez ilegales y abiertamente toleradas por el Gobierno como producto de negociaciones específicas tras las cuales con frecuencia se vislumbran intereses monetarios.

El texto muestra cómo la represión refuerza las fronteras entre las clases sociales, estigmatizando fuertemente a los pobres y dejando en la opacidad la adicción de quienes pudieron pagar por ello, algo que conduce a una inversión de los términos en que solemos pensar los silencios de la historia y las ausencias en los relatos históricos:

En los testimonios judiciales se encuentran las voces de los sujetos y se puede discernir su nivel o estatus social. Quienes fueron protegidos, o pagaron un precio alto por su libertad, no dejaron huellas en los documentos. Ellos no "pueden hablar". Quienes sí "hablan" son aquellos atrapados por el sistema, sentenciados por delitos menores $y$ mayores; por lo común gente humilde, gente de campo y socialmente marginada (p. 222).

Este capítulo, además, permite conocer uno más de los estigmas que en su tiempo pesaron sobre la comunidad china de aquellas ciudades, y señala la forma en que sus descendientes rechazan ese señalamiento. Se suma, en ese sentido, a esfuerzos recientes como los de Miguel Lisbona Guillén para rescatar del silencio la historia de los inmigrantes chinos en México (Lisbona Guillén, 2014).

Sigue a este capítulo el estudio de Pedro Cázares Aboytes sobre "Sectores populares y criminalidad en el Mazatlán 
porfiriano: prácticas y discursos", que ofrece un panorama sobre el puerto de Mazatlán en el cambio del siglo XIX al XX, trazando un breve esbozo de su vida social, subrayando la preocupación de sus élites por la seguridad y mostrando los delitos y crímenes más comunes en ese tiempo, de acuerdo con lo que arroja la exploración de los principales archivos locales, así como de los principales periódicos que circulaban en esa ciudad en la época. Se trata de una exploración que permite al autor afirmar la diversificación del delito durante uno de los periodos estereotípicamente pensados bajo el signo del orden, como es el Porfiriato. Con la riqueza de las fuentes movilizadas, pero aún poco explotadas, es de desear que el autor tenga ocasión de profundizar en las distintas aristas del tema.

El texto de Carlos Aguirre, "Apogeo, crisis y transformación del panóptico iberoamericano: apuntes para la historia de un modelo arquitectónico" es un estudio que sobresale del conjunto por su carácter comparativo, y lleva al lector a un universo geográfico más amplio ofreciendo un panorama que contempla las dos márgenes del Atlántico. Esta aproximación a la suerte corrida por el modelo de Jeremy Bentham en Iberoamérica es un trabajo de historia institucional y arquitectónica que se pregunta por los edificios en que se materializó, sus contextos sociales, su función social y política, su declive, la transformación de su uso y su relación estrecha con la memoria de tiempos dolorosos.

En ese sentido, el texto resulta central para la problemática general del libro, pues no se mantiene en los límites comunes de la historia de las instituciones y la arquitectura, sino que interroga la relación entre las políticas de creación de espacios para la represión del delito, para la reforma moral de los infractores de la ley, la transformación histórica de esos espacios (los productos del modelo) y su potencial carácter de lugares de memoria (si bien el autor no acude a la expresión ya clásica de Pierre Nora ni a la obra de este 
historiador). El autor hace del destino de los edificios desafectados como prisiones un observatorio de las políticas de memoria o desmemoria de los Gobiernos contemporáneos en Iberoamérica y señala de paso su relación con el mundo empresarial.

El texto da cuenta también de una transformación asociada a la historia de estos edificios, pero que los rebasa con mucho: la historia del declive del modelo benthamiano, extendida ahora sobre más de ocho décadas. En su transcurso, el debate sobre la necesidad de conservar estos mudos testigos de la represión social y política se ha intensificado:

En décadas recientes han tenido lugar intensos debates sobre qué destino darle a esas construcciones, testigos de algunas de las facetas más oscuras y ruines de la historia de estas sociedades. Colectivos de derechos humanos se han enfrentado no sólo a las fuerzas del capital inmobiliario, sino también a quienes buscan ocultar y silenciar las luchas y sufrimientos de quienes fueron víctimas de los aparatos represivos policiales y penales (p. 300).

Esto es indudable por las razones que el autor explica, pero sin duda también porque la memoria -asociada estrechamente a la demanda creciente de justicia- ocupa un lugar central en la movilización social y política de nuestros tiempos y en esa medida se vuelve, como lo demostró el intenso e incansable trabajo de Paul Ricoeur, un espacio de reconstitución y salvaguarda de lo humano (Ricoeur, 2000).

En el tema de los modelos carcelarios se centra también Daniel Fessler, para trabajar el caso uruguayo, caracterizado largo tiempo por la ausencia de espacios de reclusión suficientes para dar cabida a la actividad represora del Estado: "Entre los modelos penitenciarios y la realidad: las cárceles montevideanas en el Uruguay de la 'modernización penal' (1878-1910)”. Este texto, que descansa en la exploración combinada de un conjunto de fuentes tanto inéditas 
como publicadas, reconstruye cuidadosamente los debates sobre el modelo penitenciario ideal y su confrontación con la realidad de la práctica del reclusorio. Da cuenta de la inserción plena del Uruguay en los debates internacionales de su tiempo en materia de diseño de cárceles, teniendo por marco la idea de la "modernización penal" que fue preocupación generalizada del mundo occidental de la época, cuyas élites se dividieron en opiniones sobre la forma en que los espacios de privación de la libertad de los individuos que habían quebrantado la norma contribuían menos o más a la "conversión" y la "regeneración" de dichos individuos, y a su ulterior reincorporación a la sociedad.

Del espacio carcelario, pero desde su interior y centrando la perspectiva en la dimensión individual y la experiencia de la reclusión, trata el capítulo de Jorge Alberto Trujillo Bretón, "Un joven en una jaula horrible: discursos carcelarios y tácticas utilizadas por el ciudadano norteamericano Samuel C. Graham en la penitenciaría jalisciense Antonio Escobedo para obtener su libertad, 1891", un ensayo realizado a partir del caso de un recluso estadounidense en la prisión que en su tiempo fuera considerada como la más moderna de la república mexicana.

El autor se acerca al caso de Graham, quien purgaba una pena de veinte años por homicidio, y a las tensiones reveladas en torno suyo a partir de una nota aparecida en un periódico norteamericano, la cual, sin referirse a su caso, criticaba las condiciones de una prisión mexicana y paradójicamente desató un revuelo importante en que intervinieron representantes diplomáticos y autoridades. El caso permite a Trujillo reflexionar sobre la condición de marginación redoblada por la condición de extranjero de Graham en su, sin embargo, condición simultánea de preso privilegiado, y analizar el discurso de las autoridades sobre la cárcel de Escobedo en el momento de tensión desencadenado por la nota.

\section{6}


Este ensayo, por supuesto, debe leerse en el marco del amplio y documentado trabajo del mismo autor sobre la prisión de Escobedo, cuyas tesis y hallazgos centrales en materia del funcionamiento práctico del reclusorio subyacen al capítulo quizás demasiado implícitamente, aunque se comprenda que esto pueda deberse a razones de espacio o de modestia del autor (Trujillo Bretón, 2011). La reflexión de este capítulo permite enriquecer lo que sabemos sobre las condiciones de vida de los extranjeros en México, el trato diferenciado de que fueron objeto según su origen y la percepción que de ellos tuvieron las autoridades y la sociedad, un tema que en épocas recientes ha sido objeto de sugerentes esfuerzos por parte de historiadores como Pablo Yankelevich (Yankelevich, 2014).

El tema de los espacios de detención también es central en el texto de Marco Antonio León León, "Educación, regeneración y punición para la infancia anormal: las escuelas correccionales de Talcahuano y Concepción, Chile (18961915)", un estudio acucioso sobre las tensiones conceptuales que acompañaron los esfuerzos de modernización penal con relación a la infancia a partir del análisis de dos casos. El capítulo, que muestra detalladamente el contexto local del eje Concepción-Talcahuano, su devenir económico y dificultades sociales, expone cómo las tensiones y la disyuntiva entre castigar y corregir, regenerar o segregar, se cruzan con el tema de la lábil definición de la infancia en la época, algo que es común al mundo occidental, en donde de manera general han quedado confundidas infancia delincuente e infancia desvalida, infancia peligrosa e infancia en peligro, como ilustran desde hace varios años los trabajos de Mathias Gardet sobre el caso francés (Gardet, 2015). El autor vincula el fracaso de la experiencia institucional a la "confusión conceptual y doctrinaria de las autoridades e intelectuales de entonces respecto de lo que debía entenderse por infancia, su educación y regeneración” (p. 352), y 
muestra las ambigüedades de una frontera imprecisa entre lo familiar y lo estatal en materia educativa a la vez que la normativización creciente de las conductas resultante de los procesos de modernización.

Ya para cerrarse, el libro traspone una frontera, no sólo geográfica, sino -y sobre todo- de perspectiva, para pasar a un espacio de redención del delito y de la marginalidad, a través del caso de las tropas francesas de élite conocidas como "legión extranjera", en el capítulo de Marcos Pablo Moloeznik, "Crimen y redención: la legión extranjera en la guerra de intervención francesa". Este texto reconstruye las primeras décadas de vida de dicho cuerpo y muestra la persistencia hasta nuestros días de elementos formados en el siglo XIX en las experiencias históricas de las guerras coloniales francesas y su incorporación a las tradiciones reivindicadas por este controvertido instrumento militar del poder colonial francés.

El autor subraya la importancia, dentro del imaginario asociado a este cuerpo aún en nuestros días, de la experiencia habida en territorio mexicano en los años de la intervención. Como se puede apreciar en el texto, los sujetos incorporados a este cuerpo pueden acceder a una forma de redención de su marginalidad y de sus crímenes (siendo el caso) por la vía del servicio a una patria ajena a la que rinden una cuota de sangre. Aquí el discurso no insiste en la rehabilitación, sino en la redención y, si seguimos al autor, el mecanismo institucional e ideológico alcanza plenamente sus objetivos de dotar al Estado francés de un cuerpo de altísima efectividad militar.

La riqueza del conjunto del volumen, que explora desde variadas aristas cuestiones comunes a sociedades empeñadas en seguir el paradigma de la modernización de la justicia y enfrentadas por consiguiente a sus paradojas, a sus dificultades conceptuales y prácticas, lleva a desear que se hubiera presentado de manera más sistemática una 
discusión sobre lo que se considera marginal desde una perspectiva histórica e historiográfica, uno de los grandes ejes de la compilación, cuyas premisas en muchos casos permanecen implícitas en los textos y se suponen sobreentendidas por el lector.

Lo anterior no obsta para que se agradezca el esfuerzo de producir y publicar este libro, rico en pistas de reflexión y de trabajo, que da cuenta de la complejidad de los procesos occidentales de modernización de la justicia, de sus premisas teóricas, de sus experiencias, que explora su relación con la marginalidad. Una obra que, en su conjunto, muestra las dificultades de una escritura que busca partir del reconocimiento y cuestionamiento de los estereotipos, pero también la riqueza de las fuentes para la reconstrucción de estas variadas historias. Un libro, finalmente, cuya lectura invita a insistir en la importancia de la mirada aguda y plural sobre el pasado y el presente para la construcción de una narrativa histórica que abra espacios a la memoria y combata por esa vía la marginación y el olvido.

Gardet, M. (20I5). Une histoire de la jeunesse en marge [en Bibliografía colaboración con Fabienne Waks]. París:Textuel.

Lisbona Guillén, M. (20I4). Allí donde lleguen las olas del mar... Pasado y presente de los chinos en Chiapas. México:UNAM. Loraux, N. (1985). Farons tragiques de tuer une femme. París: Hachette.

Ricoeur, P. (2000). La mémoire, I histoire, I oubli. París: Éditions du Seuil.

Trujillo Bretón, J.A. (20I I). Entre la celda y el muro: Rehabilitación social y prácticas carcelarias en la penitenciaría jalisciense Antonio Escobedo, 1844-1912. Zamora: El Colegio de Michoacán.

Yankelevich, P. (20|4). "Naturalización y ciudadanía en el México posrevolucionario". Estudios de Historia Moderna y Contemporánea de México, (48), I I3-I55. 